\title{
Collisional Ionization of Na Atoms Excited by One- and Two-Photon Absorption in $\mathrm{H}_{2}-\mathrm{O}_{2}-\mathrm{Ar}$ Flames
}

\author{
C. A. VAN DIJK* and C. TH. J. ALKEMADE $†$ \\ Fysisch Laboratorium, Rijksuniversiteit Utrecht. The Netherlands
}

\begin{abstract}
An ionization signal was detected when a flashlamp-pumped dye laser was tuned on resonance with various one- or two-photon transitions of $\mathrm{Na}$ atoms in an $\mathrm{H}_{2}-\mathrm{O}_{2}-\mathrm{Ar}$ flame of $1800 \mathrm{~K}$ at atmospheric pressure. Ionization signals were obtained by collecting the electric charge with two thin iridium probes which were immersed into the flame. Relative ionization signals were measured as a function of $\mathrm{dc}$ probe voltage, laser tuning, Na-solution concentration, and free-O, concentration. Fluorescence signals served to monitor the relative populations of the higher $\mathrm{Na}$ levels. A preliminary qualitative interpretation is given on the basis of collisional ionization from the saturated as well as the higher levels, which are mutually connected by population redistribution. With the assumption of a quasi-steady-state ionization, an effective Saha equation is derived, which connects the concentration of the atoms in the saturated level with the ionic concentration by an effective ionization constant. Values of this constant were estimated from the experiments and compared for the different excitation modi investigated. Additional one-photon wing excitation of the $3 P-5 S$ transition might play a role as an intermediate step in the ion production when the $3 P$ level is saturated. The presence of excess $\mathrm{O}_{2}$ molecules enhances the degree of ionization, whereas the addition of comparable quantities of $\mathrm{N}_{2}$ molecules does not have this effect. This is explained by the positive electron affinity of $\mathrm{O}_{2}$, which shifts the effective ionization equilibrium. The role of multiphoton processes which directly transfer $\mathrm{Na}$ atoms from the $3 S$ or $3 P$ state to the continuum state is shown to be negligible. Also the role played by the associative ionization reaction: $\mathrm{Na}(3 P)+\mathrm{Na}(3 P) \rightarrow \mathrm{Na}_{2}^{+}+$ $e^{-}$is shown to be negligible under our experimental conditions.
\end{abstract}

\section{INTRODUCTION}

As an extension of our interest in the population transfer between the higher levels of $\mathrm{Na}$ atoms in an $\mathrm{H}_{2}-\mathrm{O}_{2}$ - Ar flame [1], we also investigated the ionization from excited states, which causes a loss of neutral $\mathrm{Na}$ atoms. Earlier investigations proved the existence of metal ions in flames as a result of thermal collisions and the exponential dependence of the rate constant of ionization on the ionization energy as counted from the ground state, and on the reciprocal temperature $[2,3]$. Therefore, it is to be expected that, at a given tem. perature, the ionization rate constant will increase when a substantial fraction of the alkali atoms is brought to a higher excited state by laser irradiation.

\footnotetext{
* Present address: Michigan State University, Chemistry Building, East Lansing, MI 48824.

$\dagger$ Author to whom reprint requests should be directed.
}

Notwithstanding the known disadvantages of electrical probes at elevated pressures [4], they seem a suitable means to detect ionization signals on a microsecond time scale. Probes appear to be better suited than microwave or $R F$-resonance techniques for investigating the small flame region irradiated by a (focused) laser beam.

A number of workers have reported ionization phenomena following laser excitation and the term "optogalvanic effect" is sometimes used in this connection [5-9]. Related experiments are reported in $[1,10]$, which deal with two-photon excitation and the resulting population redistribution among the higher levels of $\mathrm{Na}$ atoms in an $\mathrm{H}_{2}-\mathrm{O}_{2}$-Ar flame.

Since our main aim was to give a preliminary, qualitative interpretation of the results, we generally restricted ourselves to checking order-of-magnitude agreement between the measurements and simplified models. 


\section{EXPERIMENTAL SETUP}

The excitation source is a flashlamp-pumped tunable dye laser with a peak power of several kilowatts [11]. Pulse duration is $1 \mu \mathrm{sec}$ and repetition rate is approximately $1 \mathrm{~Hz}$. The full width at half maximum (FWHM) of the spectral laser profile was determined to be $140 \mathrm{~m} \AA$ at $5890 \AA$; this width exceeds atomic linewidths in flames by a factor of two to three [12]. Rough tuning of the laser is carried out by tilting an interference filter; fine tuning is done with a Fabry-Pérot etalon. Dyes used are Rhodamine 6G dissolved in methanol, and a mixture of Rhodamine $6 \mathrm{G}$ and Cresylviolet dissolved in methanol.

The flame is a stoichiometric $\mathrm{H}_{2}-\mathrm{O}_{2}-\mathrm{Ar}$ flame at atmospheric pressure and a temperature of 1800 $\mathrm{K}$. The burner is of the Méker type and has a manthe flame to prevent infusion of the surrounding air. The flow system is identical to that described in [13].

The laser beam traverses the flame at a height of $10 \mathrm{~mm}$ above the combustion zone. The diameter of the cylindrical flame at this height is 15 $\mathrm{mm}$. A small part of the laser beam is split off for power monitoring. Power calibration is derived from a pyroelectric detector.

Ionization signals are detected with two iridium wires with a rectangular cross section of $0.1 \times 0.5$ $\mathrm{mm}^{2}$ which are suspended in the flame in the vicinity of the laser beam (see Fig. 1). These probes proved to be highly corrosion-resistive, even with excess $\mathrm{O}_{2}$ present in the flame, and showed no deterioration even after several hundreds of hours of operation. A voltage of $300 \mathrm{~V}$ is maintained between the probes and ionization signals appear across a load resistor which is followed by an impedance transformer.

Fluorescence light is collected by a quartz lens which images a portion of the irradiated flame volume onto the slit of a grating monochromator. With the slits set at $1 \mathrm{~mm}$, this monochromator has a bandwidth of $23 \AA$ (FWHM). A photomultiplier detects the visible and $U V$ light at the exit slit of the monochromator. The electric signals from the probes, the photomultiplier, and the power monitor are processed by gated integrators whose outputs are displayed on $x$ - $t$ recorders. The mea- surement of the total charge contained in an ionization signal is facilitated by the gated integrators and we assume that the signals measured in this way are proportional to the time-averaged density of charged species in the excited volume.

The various detector outputs can be observed two at a time on a true dual-beam oscilloscope which has a rise time of $10 \mathrm{nsec}$. The relative spectral response of the light-detection system has been determined with the aid of a calibrated tungsten-filament lamp. The bulk displacement of the flame gases during the microsecond laser pulse is of the order of $10 \mu \mathrm{m}$.

\section{RESULTS AND DISCUSSION}

\subsection{General}

The laser was tuned to several one- or two-photon transitions of sodium and the resulting ionization signals were detected as a function of, e.g., probe voltage, laser tuning, sodium concentration, and free molecular oxygen concentration of the flame. The simultaneous recording of ionization and fluorescence signals facilitates a direct comparison.

Saturation and saturation broadening were observed with both one- and two-photon excitation. Detailed information concerning the observed saturation may be found in [10] .

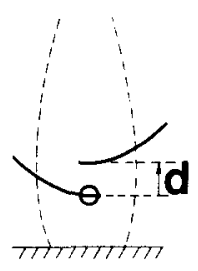

a

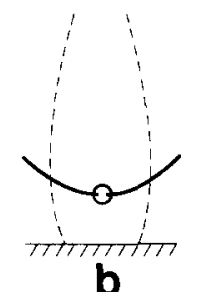

b

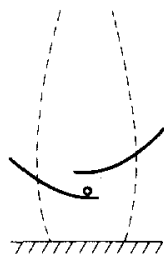

C
Fig. 1. Probe and laser-beam configurations used. Small circle: cross section of the laser beam whose diameter is 3 $\mathrm{mm}$ approximately if laser is not focused; heavy lines: probes; dashed lines: outline of the inner flame; hatched area: burner head. Fluorescence is detected at right angles to the laser beam and the field of view of the detection system is kept sufficiently clear by bending the probes slightly. (a) Unfocused beam striking the negative probe. (b) Unfocused beam striking both probes. (c) Focused beam, with diameter of $0.1 \mathrm{~mm}$ approximately, bypassing the probes. 
From the fact that no variation of the fluorescence signals was observed whether the probes were in the immediate vicinity of the excited volume or not, we infer that the population distribution of the excited levels was not noticeably affected by the presence of the probes. From the ionization signals obtained with a blank solution nebulized into the flame and the laser on resonance with various one- or two-photon transitions, we conclude that the contribution of natural flame electrons and impurity sodium ions to the ionization signals is negligible. A sodium solution concentration of $2560 \mu \mathrm{g} / \mathrm{mliter}$ was used throughout the experiments with the exception of those reported in Section 3.4. In most cases, we used the $U V$ line of the $4 P-3 S$ transition to monitor the population of the higher levels, primarily because of the excellent signal-to-noise ratio of this line.

\subsection{Probes}

Because of the irregular shape of the probes, we restrict ourselves to a phenomenological description and qualitative considerations; probe theories at elevated pressures are complex, even for probes with spherical or cylindrical shapes [4].

We briefly investigated the behavior of the probe signals when the probes were in different configurations, both with respect to each other and with respect to the excited volume. In the configuration of Fig. 1(a), the laser beam has a diameter of approximately $3 \mathrm{~mm}$ (unfocused) and strikes the negative probe, the latter being at a distance of $10 \mathrm{~mm}$ from the positive probe, which is well outside the laser beam. The time-integrated ionization current as a function of the distance $d$ between the probes is shown in Fig. 2; the probe which is exposed to the laser beam, being tuned to the $\mathrm{Na}\left(3 S-3 P_{3 / 2}\right)$ transition, has a fixed position with respect to this beam and is at the same potential as the burner head. When the fixed probe is negative (with respect to the movable probe), the measured charge is almost independent of $d$, but when the fixed probe is positive, a considerable drop occurs with increasing $d$. This observation shows that the current is limited by the sodium ions, which obviously move too slowly to cross the space between the probes within the time that

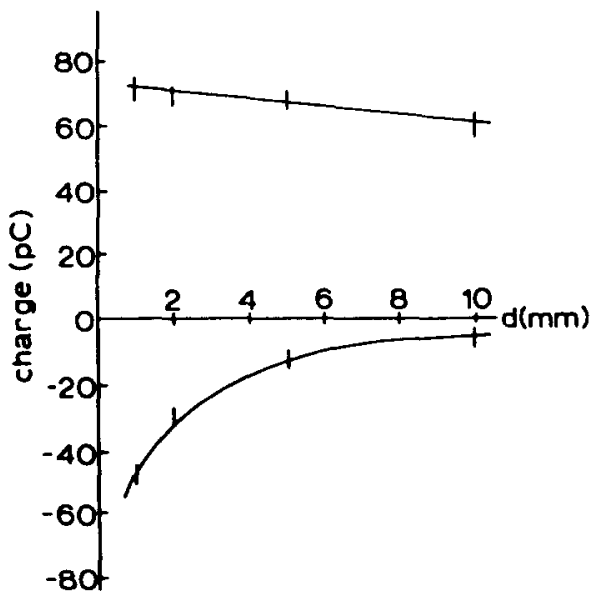

Fig. 2. Ionization charge as a function of the distance $d$ between the probes, measured with the probe configuration of Fig. 1(a), where the lower probe is kept in a fixed position. The upper curve is obtained with the lower probe negative and the lower curve is obtained with the polarity of the probes reversed. The laser was tuned to the $3 S-3 P_{3 / 2}$ transition of $\mathrm{Na}$ and the irradiance was $7 \times 10^{4}$ $\mathrm{W} / \mathrm{cm}^{2}$

the gate of the integrator is open $(\cong 10 \mu \mathrm{sec})$; recombination might also prevent the sodium ions from reaching the negative probe. If we assume the mobility of the sodium ions to be of the order of $2 \mathrm{~cm}^{2} \mathrm{sec}^{-1} \mathrm{~V}^{-1}$, as is found in flames at atmospheric pressure [14], we obtain $60 \mu \mathrm{m}$ for the distance crossed within $10 \mu \mathrm{sec}$ at a field strength of $300 \mathrm{~V} / \mathrm{cm}$. This distance is about twice the RMS distance crossed by these ions under the influence of diffusion.

The comparison of the results for the probe configurations of Fig. 1(a) and (b) offers the possibility of observing the effects of electron capture by $\mathrm{O}_{2}$ in an oxygen-rich flame. Both kinds of negative charge carriers are able to reach the positive probe in the case of Fig. 1(b), whereas only the faster moving electrons can reach this probe in the case of Fig. 1(a) (see Section 3.5).

The charge-voltage characteristic ( $\mathrm{cvc}$ ) of the configuration of Fig. 1(a) was found to have a nearly constant slope of one on double-logarithmic scales; the laser was tuned to the $3 S-3 P_{3 / 2}$ transition of $\mathrm{Na}$ and the irradiance was $18 \mathrm{~kW} / \mathrm{cm}^{2}$, which is far above the estimated saturation value of $22 \mathrm{~W} / \mathrm{cm}^{2}$ [15]. We infer that the plasma between the probes behaves almost ohmically; from 
the slope of the cvc and the average current, we estimate the resistance of the plasma to be of the order of $1 \mathrm{M} \Omega$.

For two-photon excitation, the laser beam was focused down to a spot with a diameter of approximately $100 \mu \mathrm{m}$. To obtain an adequate signalto-noise ratio it appeared necessary to locate the probes in the immediate vicinity of the beam waist but in such a manner as not to obstruct the detection of fluorescence light and avoid as much as possible the reflections of laser light on the probes; these reflections may cause considerably more background in the fluorescence signal with a focused beam than with an unfocused beam. A geometry which reasonably satisfies the above requirements is shown in Fig. 1(c).

A time-resolved ionization signal resulting from excitation of the $3 S-3 D$ two-photon transition is given in Fig. 3. The ionization pulse is seen to decay somewhat slower than the laser pulse, probably as a result of the slowing down of the recombination rate after the cessation of the laser pulse.

With the probes at a distance of approximately $1 \mathrm{~mm}$ from each other, we observed that bias voltages in excess of approximately $400 \mathrm{~V}$ produced breakdown of the flame plasma between the probes; breakdown currents were in the order of $100 \mathrm{~A}$.

\subsection{Fluorescence Excitation and Ionization Profiles}

Profiles of ionization and fluorescence signals are obtained when the laser is varied across an atomic resonance (see [1]).

Figure 4 shows the fluorescence-excitation profile and ionization profile of the $\mathrm{Na}\left(3 S_{-}-3 P_{3 / 2}\right)$ transition, obtained with the probe configuration of Fig. 1(b). The width of the ionization profiles is seen to be significantly different from the width of the fluorescence profile, and both profiles appear to be broader than $140 \mathrm{~m} \AA$, which is the width of the spectral-laser profile. As shown elsewhere [10], the broadening of the fluorescenceexcitation profile is due to the dependency of the absorption coefficient on the laser irradiance as well as on the laser tuning (so-called saturation broadening).

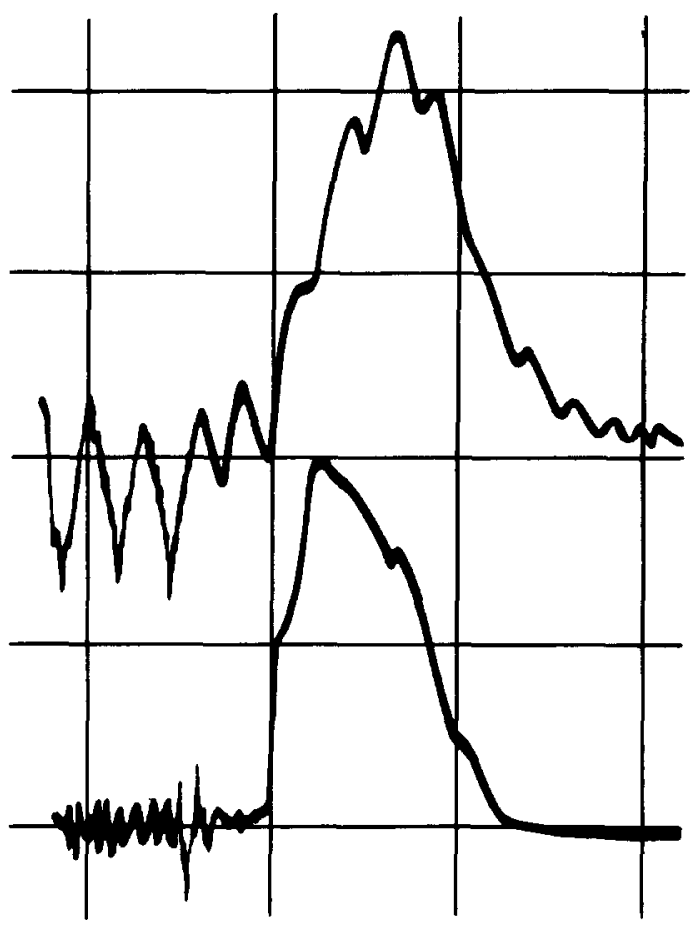

Fig. 3. Time-resolved pulse shapes obtained with $3 S-3 D$ two-photon excitation and the probe configuration of Fig. 1(c). A Tektronix 556 dual-beam oscilloscope was used, with the time base set to $1 \mu \mathrm{sec} / \mathrm{div}$. Lower trace: laser pulse, irradiance $4 \times 10^{3} \mathrm{~kW} / \mathrm{cm}^{2}$. Upper trace: ionization signal as detected by the probes. The ringing preceding the pulses is due to pickup of RF noise which is produced by the spark gap which fires the laser.

Fluorescence excitation and ionization profiles of two-photon transitions, obtained with the probe configuration of Fig. 1(c), show qualitatively the same behavior as the profiles of Fig. 4 . Beam focusing and appropriate tuning also produce excitation of the $3 P_{1 / 2}-5 S$ and $3 P_{3 / 2}-5 S$ one-photon transitions; the profiles obtained with the latter excitations show a similar difference in width between the ionization and excitation profiles as found in Fig. 4.

In all profile measurements, whether they are done with one- or two-photon excitation and regardless of the probe configuration, laser power, or fluorescence monitor, the width of the ionization profile exceeds that of the fluorescence-excitation profile. This difference in width may be explained as follows: we assume the ionization to proceed 




Fig. 4. Simultaneously recorded ionization profile (upper curve) and fluorescenceexcitation profile (lower curve) obtained with the laser being tuned across the $3 S-3 P_{3 / 2}$ transition. Probe configuration was that of Fig. 1(b); irradiance of the pulsed laser: $5 \times 10^{4} \mathrm{~W} / \mathrm{cm}^{2}$; fluorescence monitor: $4 P-3 S$ transition. The dashed curve represents the square-root of the fluorescence curve. Both curves are normalized to a peak value of unity.

according to the single-step endoergic-ionization process

$\mathrm{Na}^{*}+Z \rightarrow \mathrm{Na}^{+}+e^{-+} Z$

where $\mathrm{Na}^{*}$ represents an excited $\mathrm{Na}$ atom whose number density, $\left[\mathrm{Na}^{*}\right]$, is enhanced appreciably above its thermal value as a result of laser excita. tion; $Z$ denotes an arbitrary flame-gas atom or molecule. The excited state involved is not necessarily limited to the directly laser-excited state. Due to collisional redistribution of the population of the $\mathrm{Na}$ levels [1], other levels below and above the directly excited state might also contribute to the ionization. If we assume that the populations of those excited levels $\left(\mathrm{Na}^{*}\right)$ which occur in Reaction (1) are proportional to the population of the laser-excited level, $\left[\mathrm{Na}^{\phi}\right]$, we may express the ionization rate $R_{i}{ }^{\phi}$ (number of ionizing collisions per second and per $\mathrm{cm}^{3}$ ) by

$R_{i}^{\phi}=k_{i}^{\phi}\left[\mathrm{Na}^{\phi}\right]$

where $k_{i}^{\phi}$ is a pseudo-first-order ionization rate constant (in $\sec ^{-1}$ ) referring to the laser-excited level population.

Recombination is assumed to occur through the reverse single-step process

$\mathrm{Na}^{+}+e^{-}+Z \rightarrow \mathrm{Na}+Z$ where the neutral $\mathrm{Na}$ atom may be in any (excited or ground) state. We have neglected radiative recombination processes, because at atmospheric pressure, collisions may be assumed to be dominant. The rate of recombination $R_{r}$ can be described by

$R_{r}=k_{r}(T)\left[\mathrm{Na}^{+}\right]\left[e^{-}\right]$.

Here we have assumed that the pseudo-secondorder recombination rate constant $k_{r}(T)$ (in $\mathrm{cm}^{3}$ $\sec ^{-1}$ ) is not affected by the laser excitation of the neutral atoms and equals its thermal value at flame temperature $T$.

If the Reactions (1) and (3) equilibrate each other during the laser pulse, we have $R_{i}{ }^{\phi}=R_{r}$, from which we obtain an effective Saha equation

$\left[\mathrm{Na}^{+}\right]\left[e^{-}\right] /\left[\mathrm{Na}^{\phi}\right]=k_{i}^{\phi} / k_{r}(T) \equiv K_{i}{ }^{\phi}$.

$K_{i}{ }^{\phi}$ is defined here as an effective ionization constant corresponding to the effective ionization energy $E_{i}{ }^{\phi}$ (see below). We assume the partially ionized flame volume to be electrically neutral as a whole, thus $\left[\mathrm{Na}^{+}\right]=\left[e^{-}\right]$, which gives, upon application to Eq. (5)

$\left[\mathrm{Na}^{+}\right]=\left(K_{i}^{\phi}\left[\mathrm{Na}^{\phi}\right]\right)^{1 / 2}$.

If the population of the fluorescence monitor level $\left[\mathrm{Na}^{m}\right]$ is assumed to be proportional to that 
of the laser-excited level $\left[\mathrm{Na}^{\phi}\right]$, it follows that the ion concentration is proportional to the square root of the population of the monitor level; a behavior which is in semiquantitative agreement with the experimental profiles of Fig. 4.

If we suppose that the majority of the excited levels lying between the laser-excited level and the ionization continuum are in partial Boltzmann equilibrium at the flame temperature $T[1], k_{i}{ }^{\phi}$ might be conceived as a quasi-thermal ionization rate constant, $k_{i}{ }^{\phi}(T)$. In analogy with the experimental behavior of the true thermal ionization rate constant we might expect $k_{i}{ }^{\phi}(T)$ to contain an activation energy factor $\exp \left[-E_{i}{ }^{\phi} / k T\right]$, where $E_{i}{ }^{\phi}$ is the ionization energy as counted from the laser-excited level and $k=$ Boltzmann's constant. The latter level acts as a quasi-ground level because of the saturation. The effective ionization constant $K_{i}{ }^{\phi}$ in Eq. (5) might then be identified with a quasithermal ionization constant $K_{i}{ }^{\phi}(T)$ which exceeds the true thermal Saha constant, $K_{i}(T)$, by a factor of the order of $\exp \left[E_{\operatorname{exc}} \phi / k T\right]$, where $E_{\text {exc }}{ }^{\phi}=$ $E_{i}-E_{i}^{\phi} ; E_{i}=$ ionization energy as counted from the ground level; $E_{\mathrm{exc}} \phi=$ excitation energy of the laser-excited level. (We have ignored complications which might arise from the appearance of partition functions in the statistical-mechanical expression of the true thermal Saha constant.)

\subsection{Dependence of the Ionization Signal on the $\mathrm{Na}$ Solution Concentration}

The equilibrium degree of ionization of a given species is known to depend on the total concentration of that species in the flame. In order to observe the behavior of the degree of ionization under conditions of laser irradiation, the laser was tuned to the $3 S-3 P_{3 / 2}$ transition of sodium and the probe configuration of Fig. 1(a) was used. Different Na solution concentrations were sprayed into the flame and the resulting ionization signals divided by the corresponding $\mathrm{Na}$ solution concentrations are plotted against those concentrations (see Fig. 5). With increasing concentrations, the value of the slope of the double-logarithmic curve is seen to approach the value -0.5 . This behavior is consistent with the effective Saha equation (6).
To show this, we define the degree of ionization $\beta$ :

$\beta \equiv\left[\mathrm{Na}^{+}\right] /[\mathrm{Na}]_{t}$

where $[\mathrm{Na}]_{t}$ denotes the total concentration of free sodium atoms and ions

$[\mathrm{Na}]_{t} \equiv\left[\mathrm{Na}^{0}\right]+[\mathrm{Na} \phi]+\left[\mathrm{Na}^{+}\right]$

and $\left[\mathrm{Na}^{0}\right]$ and $\left[\mathrm{Na}^{\phi}\right]$ are the concentrations of sodium atoms in the ground- and laser-excited states; we assume that the concentration of the atoms in other states is negligible. $\beta$ is proportional to the ordinate value of Fig. 5 if we assume the probe signal to be proportional to $\left[e^{-}\right]=\left[\mathrm{Na}^{+}\right]$ and $[\mathrm{Na}]_{t}$ to be proportional to the $\mathrm{Na}$ solution concentration. The latter proportionality holds, in good approximation, also when $\mathrm{NaOH}$ is formed, if the fraction of ionized sodium is small. We define the relative population of the saturated level, $\epsilon$, as follows:

$\epsilon \equiv \frac{\left[\mathrm{Na}^{\phi}\right]}{\left[\mathrm{Na}^{\phi}\right]+\left[\mathrm{Na}^{0}\right]}$.

From Eqs. (6)-(9), we obtain

$\frac{\beta^{2}}{1-\beta}=\frac{\epsilon K_{i}^{\phi}}{[\mathrm{Na}]_{t}}$

For $[\mathrm{Na}]_{t} \gg K_{i}^{\phi}$, we have $\beta \ll 1$ and consequently

$\beta \cong\left(\frac{\epsilon K_{i}^{\phi}}{[\mathrm{Na}]_{t}}\right)^{\frac{1}{2}}$.

Thus the slope of -0.5 of the asymptote in Fig. $5(\mathrm{a})$ indicates that $[\mathrm{Na}]_{t}$ is such that $\beta \ll 1$ or $\left[\mathrm{Na}^{+}\right] \ll[\mathrm{Na}]_{t}$. For $[\mathrm{Na}]_{t} \ll \epsilon K_{i}^{\phi}$, we expect from Eq. (10) that $\beta$ tends to unity and thus becomes independent of the $\mathrm{Na}$ concentration. We note that Eq. (10) remains valid also when $\mathrm{Na}$ compounds are formed, if we use the same definition of $[\mathrm{Na}]_{t}$ as in Eq. (8).

A curve similar to that obtained with one-photon excitation was obtained with two-photon excitation and is shown in Fig. 5(b). As the latter curve also exhibits an asymptotic slope of -0.5 , it might 


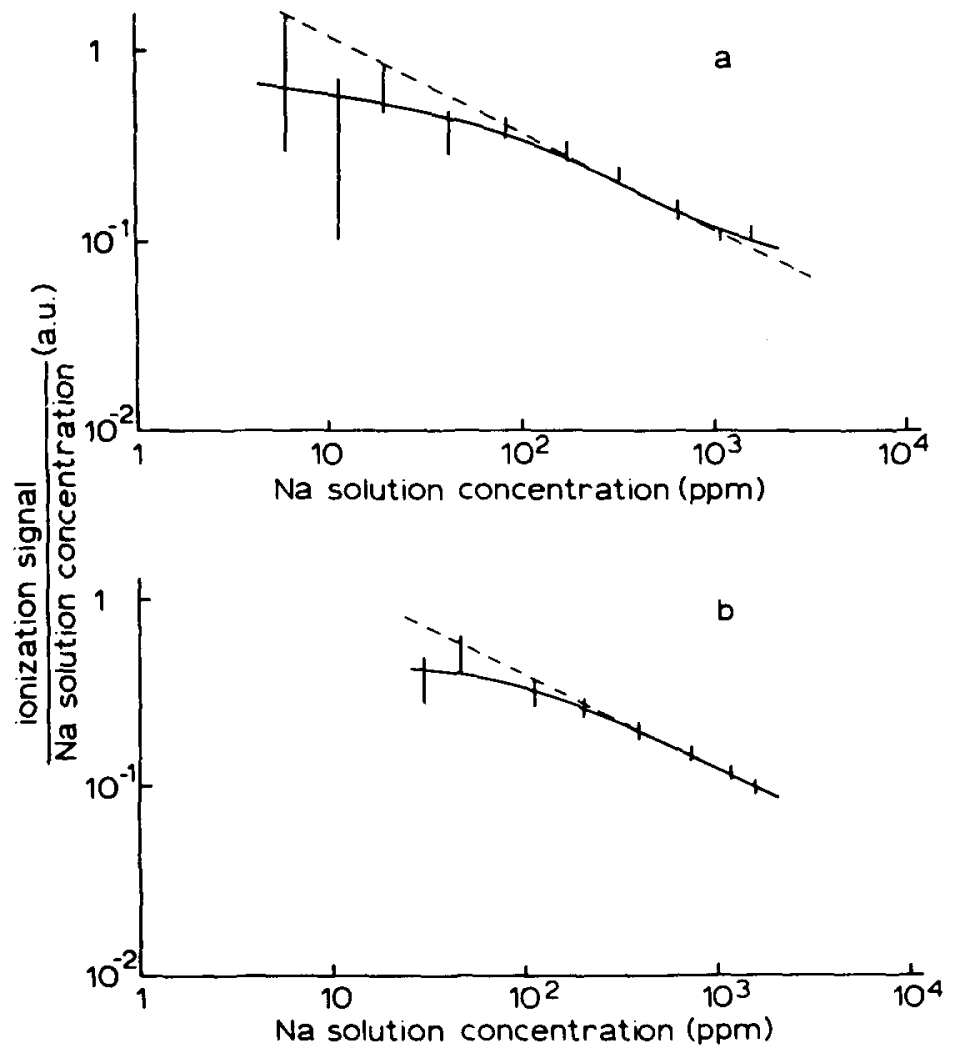

Fig. 5. Ratio of ionization signal to sodium solution concentration plotted against the sodium solution concentration (in $\mathrm{ppm}=\mu \mathrm{g} / \mathrm{mliter}$ ) on double-logarithmic scales. The dashed line has the theoretically expected slope of -0.5 for large sodium solution concentrations at which the degree of ionization is small. (a) Laser tuned to the $3 S-3 P_{3 / 2}$ transition; probe configuration of Fig. 1(a); irradiance: $4 \times 10^{4} \mathrm{~W} / \mathrm{cm}^{2}$. (b) Laser tuned to the $3 S-5 S$ two-photon transition; probe configuration of Fig. 1(c); irradiance: $9 \times 10^{3} \mathrm{~kW} / \mathrm{cm}^{2}$.

be inferred that, even with two-photon excitation, the degree of ionization is small for concentrations in excess of $100 \mu \mathrm{g} / \mathrm{mliter}$ approximately.

From Eq. (10), we conclude that the transition from the horizontal asymptote at low $\mathrm{Na}$ concentrations to the asymptote with slope -0.5 at high concentrations is marked by $[\mathrm{Na}]_{t} \cong \epsilon K_{i}^{\phi}$. In our case, $\epsilon$ will be of the order of 0.5. If partial Boltzmann equilibrium would exist for all or most of the levels above the laser-excited level, $K_{i} \phi(T)$ is expected to be of the order of $K_{i}(T) \exp \left[+E_{\mathbf{e x c}} \phi / k T\right]$. Thus we expect $K_{i}^{\phi}(T) \cong 4 \times 10^{11} \mathrm{~cm}^{-3}$ and $3 \times 10^{17} \mathrm{~cm}^{-3}$ at $T=1800 \mathrm{~K}$ with the $3 P$ level and the $5 S$ level, respectively, being taken as the quasi-ground level. It appears from Fig. 5(a) that the transition between the low-concentration and high-concentration asymptotes occurs at a sodium solution concentration of approximately $100 \mu \mathrm{g} / \mathrm{mliter}$ which corresponds to a value of $[\mathrm{Na}]_{t}$ of the order of $10^{12} \mathrm{~cm}^{-3}$ [16]. This outcome seems to agree roughly with the value of $K_{i}{ }^{\phi}(T)$ estimated above for the $3 P$ level. However, the observation from Fig. 5(b) that for the $5 S$ level, the transition between the two asymptotes occurs approximately at the same concentration is at variance by six orders of magnitude with the above estimate of $K_{i}{ }^{\phi}(T)$ for the $5 S$ level. We must thus conclude that the above quasi-thermal model for collisional ionization from saturated levels is an oversimplified one. Complications that may pre- 
clude a quantitative application of this model are:

i) Absence of a quasi-Boltzmann equilibrium for the levels above the saturated $3 P$ or $5 S$ level.

ii) In the case of $3 P$-level saturation, the ionization rate might be enhanced by collisional ionization from the higher $5 S$ level which could be populated through additional one-photon laser excitation in the collisionally broadened wing of the $3 P-5 S$ transition (see also Section 3.6).

iii) The gain in activation energy factor for collisional ionization from the $5 S$ level when compared to that from the lower $3 P$ level might be partly offset by a possible decrease in ionization cross section with increasing principal quantum number $n$. It is noted that for the quenching of higher excited sodium states by various gases, a maximum occurs in the cross sections with increasing $n[17,18]$. Gounand et al. $[19,20]$ have argued, on the basis of theoretical work by Smirnov [21], that the ionization cross sections are small compared with the quenching cross sections.

iv) The ionization- and fluorescence-pulse measurements are averages over time as well as over space, whereas the relative population of the saturated level, $\epsilon$, and the degree of ionization, $\beta$, vary with place as well as with time.

v) Ambipolar diffusion of the $\mathrm{Na}^{+}$ions out of the focused laser beam used in the two-photon excitation of the $5 S$ level might depress the (average) value of $\left[\mathrm{Na}^{+}\right]$. The diffusion coefficient $D$ for $\mathrm{Na}$ atoms or ions in flames as used by us is about 10 $\mathrm{cm}^{2} \mathrm{sec}^{-1}$ [22]. Since the ambipolar diffusion coefficient is twice that of the corresponding ion, we estimate that a sodium ion covers an RMS distance $\left(\overline{r_{\perp}^{2}}\right)^{1 / 2}=(8 D t)^{1 / 2} \approx 100 \mu \mathrm{m}$ perpendicular to the beam axis during the time $t$ of the laser pulse $(=1 \mu \mathrm{sec})$; the diameter of the focused beam is of the same order of magnitude. One might thus expect a loss in ion density due to ambipolar diffusion here [23]. Ambipolar diffusion in the case of one-photon excitation of the $3 P$ level can, however, be neglected, as focusing was not applied here.

vi) The expected formation of $\mathrm{NaOH}$ might reduce the value of $[\mathrm{Na}]_{t}\left(\equiv[\mathrm{Na}]+\left[\mathrm{Na}^{+}\right]\right)$by one order of magnitude.

\subsection{Dependence of the Ionization and Fluore- scence Signals on the Free Oxygen Concentration of the Flame}

Since molecular oxygen is known to have a positive electron affinity [24], it seems interesting to investigate the behavior of the ionization and fluorescence signals as a function of the free oxygen concentration in the flame. This experiment was done with the laser tuned to the $3 S$ $3 P_{3 / 2}$ transition and the probe configuration of Fig. 1(b). In Fig. 6(a) we plotted the observed ratio of ionization signal to fluorescence signal (from the monitor level $\mathrm{Na}^{m}$ ) as a function of the calculated free- $\mathrm{O}_{2}$ concentration in the flame. We assume this ratio to be proportional to $\left[\mathrm{Na}^{+}\right] /$ $\left[\mathrm{Na}^{m}\right]$, which in turn can be used as a relative measure for the degree of ionization, $\beta$, under saturation conditions, since we observed that $\beta \ll$ 1 in the stoichiometric point and at the high sodium solution concentration $(2560 \mu \mathrm{g} /$ mliter $)$ utilized. Thus the behavior of the curve of Fig. 6(a) might be explained as an increase of $\beta$ with increasing $\mathrm{O}_{2}$ concentration. Since $\beta$ increases with the effective ionization constant $K_{i}{ }^{\phi}$ [see Eq. (11)], we infer that the addition of free $\mathrm{O}_{2}$ to the flame results in an increase of $K_{i}{ }^{\phi}$. An enhanced degree of ionization for excited $\mathrm{Na}$ atoms colliding with $\mathrm{O}_{2}$ was also suggested in [15] to explain the enhanced decay rate of the time-resolved fluorescence signal from the $3 P$ level when the $\mathrm{O}_{2}$ concentration in the flame was increased. A similar change-be it less pronounced-was found by us in the time-resolved fluorescence signal of the $4 P-3 S$ transition [see insert of Fig. 6(a)] upon the addition of $\mathrm{O}_{2}$ to the flame.

We note that a reduction of $[\mathrm{Na}]_{t}$ by the formation of $\mathrm{NaO}_{2}$ and $\mathrm{NaOH}$ might also (partly) explain the increase of $\beta$ with increasing $\mathrm{O}_{2}$ concentration [cf., Eq. (11)] .

In order to explain semiquantitatively the effect of $\mathrm{O}_{2}$, consider the following reactions

$$
\begin{aligned}
& \mathrm{Na}^{\phi}+\mathrm{O}_{2} \leftrightharpoons \mathrm{Na}^{+}+\mathrm{O}_{2}^{-} \\
& \mathrm{O}_{2}^{-}+Z \leftrightharpoons \mathrm{O}_{2}+e^{-}+Z \\
& \mathrm{Na}^{\phi}+\mathrm{O}_{2} \leftrightharpoons \mathrm{Na}^{+}+e^{-}+\mathrm{O}_{2}
\end{aligned}
$$





Fig. 6. Effect of free $\mathrm{O}_{2}$ and $\mathrm{H}_{2}$ molecules on the ionization with different probe configurations. The laser was tuned to the $3 S-3 P_{3 / 2}$ transition; irradiance: $5 \times 10^{4}$ $\mathrm{W} / \mathrm{cm}^{2}$; fluorescence monitor: $4 P-3 S$ transition. (a) Ratio of the ionization signal to the fluorescence signal plotted against the partial pressures of free oxygen and free hydrogen. Probe configuration used was that of Fig. 1(b). Also shown are the fluorescence pulse shapes ob- tained at two different partial $\mathrm{O}_{2}$ pressures: the left one close to the stoichiometric point, the second one at a partial $\mathrm{O}_{2}$ pressure of several percent. The former pulse reproduces the laser pulse whereas the latter does not. Time base is $1 \mu \mathrm{sec} /$ div. (b) Ratio of the ionization signal squared to the fluorescence signal is plotted against the partial pressures of free oxygen and free hydrogen. Data were those used for Fig. 6(a). (c) Ratio of the ionization signal to the fluorescence signal is plotted against the partial pressures of free oxygen and free hydrogen. The probe configuration used was that of Fig. 1(a).

where $Z$ is an arbitrary flame-gas atom or molecule. If each one of these reactions is equilibrated, we obtain, using $\left[\mathrm{Na}^{+}\right]=\left[e^{-}\right]+\left[\mathrm{O}_{2}^{-}\right]$and eliminating $\left[e^{-}\right]$and $\left[\mathrm{O}_{2}^{-}\right]$through the appropriate Saha laws,

$\left[\mathrm{Na}^{+}\right]^{2} /\left[\mathrm{Na}^{\phi}\right]=\left\{1+\left[\mathrm{O}_{2}\right] / K_{b}(T)\right\} K_{c}{ }^{\phi}$,

with $K_{a}{ }^{\phi} K_{b}(T)=K_{c}{ }^{\phi}$. Here $K_{a}{ }^{\phi}$ and $K_{c}{ }^{\phi}$ are the effective ionization constants of the Reactions (12a) and (12c), respectively, under saturation conditions, defined in a similar way as $K_{i}{ }^{\phi}$ in Eq. (5); $K_{b}(T)$ is the equilibrium constant of Reaction (12b), which is not affected by the saturation. According to Eq. (13), it might be of interest to plot the ratio of the square of the ionization signal $\left(I^{2}\right)$ to the fluorescence monitor signal $(F)$ against the calculated free- $\mathrm{O}_{2}$ concentration [see Fig. $6(\mathrm{~b})$ ] . The qualitative agreement with Eq. (13) is apparent: the ratio $I^{2} / F$ increases roughly linearly with free- $\mathrm{O}_{2}$ concentration. We note that Eq. (13) remains strictly valid when $\mathrm{NaOH}$ or $\mathrm{NaO}_{2}$ is formed.

A closer analysis of the influence of oxygen on the ionization and fluorescence signals should take into account: i) the possible contribution of the process: $\mathrm{Na}^{\phi}+\mathrm{OH} \rightarrow \mathrm{Na}^{+}+\mathrm{OH}^{-}$to the ionization of sodium; ii) the effect of wing excitation of the higher levels (see Section 3.6); iii) $\mathrm{O}^{-}$formation; iv) the effect of excess $\mathrm{O}_{2}$ on the flame temperature; and v) the quenching of the fluorescence by $\mathrm{O}_{2}$. Capture of free electrons by $\mathrm{O}_{2}$ molecules might explain the different dependence of the ratio $I / F$ on the calculated free- $\mathrm{O}_{2}$ concentration as shown in Fig. 6(c) for the probe configuration of Fig. 1(a) with the positive probe at a distance of several millimeters from the illuminated flame region. The flattening of the curve of Fig. 6(c) ob- 
served at increasing $\mathrm{O}_{2}$ concentration is possibly due to the current-limiting effect of the slow $\mathrm{O}_{2}{ }^{-}$ ions, which might have captured a substantial fraction of the electrons.

In Fig. 7 the ratio of the ionization signal to the fluorescence monitor signal is plotted against the concentration of $\mathrm{N}_{2}$ molecules added to the flame. In contrast to the electron affinity of $\mathrm{O}_{2}$, the electron affinity of $\mathrm{N}_{2}$ is negative, so that no effect on the degree of ionization is expected upon addition of $\mathrm{N}_{2}$, in agreement with the observation in Fig. 7.

\subsection{Dependence of the Ionization Signal on the Laser Excitation Mode}

The presumed exponential dependence of the effective ionization constant $K_{i}^{\phi}$ on the ionization energy $E_{i}{ }^{\phi}$ as counted from the laser-excited level (see Section 3.3) was tested by measuring the relative ionization signal $I$ with the laser being tuned successively to the $3 S-3 P_{\mathbf{3} / \mathbf{2}}, 3 S-5 S$, and $3 S-4 D$ transitions. These measurements were done in the stoichiometric flame at a constant irradiance of $9 \times 10^{3} \mathrm{~kW} / \mathrm{cm}^{2}$ and with the probe configuration of Fig. 1(c). Estimates of the relative populations, $\left[\mathrm{Na}^{\phi}\right]$, of the laser-excited levels were obtained from fluorescence measurements and the known Einstein transition probabilities (see [25] for details). Assuming that these (nearly) saturated levels served as quasi-ground-state levels, we calculated relative $K_{i}{ }^{\phi}$ values from the relative $I$ and $\left[\mathrm{Na}^{\phi}\right]$ values by using Eq. (6).

The values of $K_{i}{ }^{5 S}$ and $K_{i}{ }^{4 D}$ appeared to be of the same order of magnitude, as was expected from the small difference of $0.16 \mathrm{eV}$ between the $E_{i}{ }^{\phi}$ values for these levels. However, $K_{i}{ }^{3 P} / K_{i}{ }^{5 S}$ appeared to be larger by four orders of magnitude than could be expected from the difference of 2.0 $\mathrm{eV}$ between the corresponding $E_{i}{ }^{\phi}$ values at $T=$ $1800 \mathrm{~K}$. This discrepancy might be (partly) explained by supposing that in the case of the $3 P$. excitation mode, additional one-photon excitation in the collisionally broadened wing of the $3 P-5 S$ transition occurred that raised $\left[\mathrm{Na}^{5 S}\right]$ to about one hundredth of $\left[\mathrm{Na}^{3 P}\right]$. This assumption is supported by the observed relative fluorescence signals from the $3 P$ and $4 P$ levels (see [25] for a more detailed discussion).

It should be noted that the ratio of $K_{i}{ }^{\phi}$ values obtained in this experiment when the laser was tuned to the $3 P$ and $5 S$ level, respectively, cannot be directly compared with the corresponding ratio estimated from the experiments described in Section 3.4. In the former experiment, a focused laser beam was used, in contrast to the experiments described in Section 3.4. This difference might have consequences because of the effect of ambipolar diffusion on the ionic densities (cf., Section 3.4).

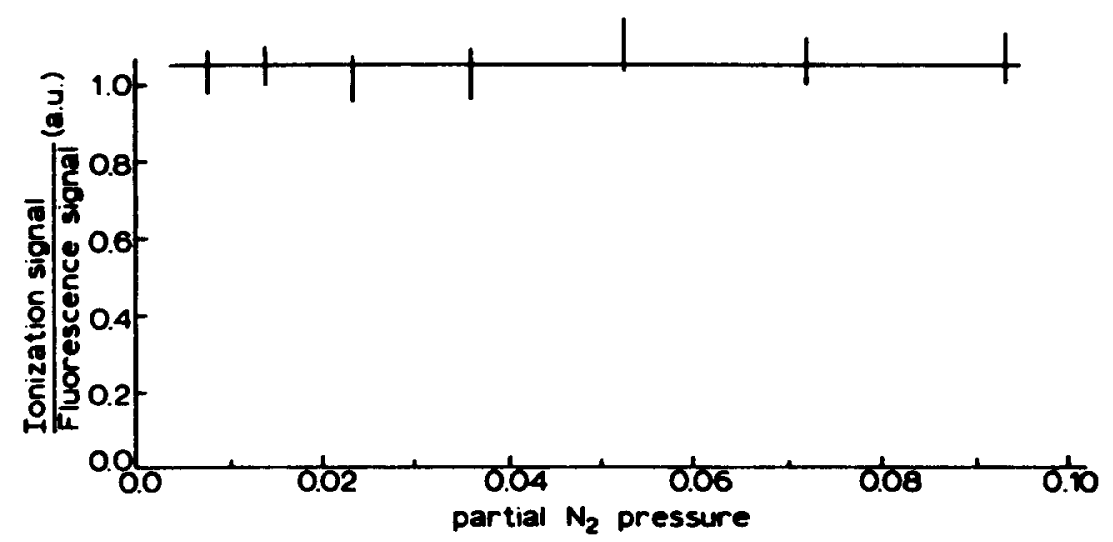

Fig. 7. Ratio of ionization signal to fluorescence signal is plotted against partial pressure of nitrogen added to the flame. The probe configuration used was that of Fig. 1 (a); the laser was tuned to the $3 S-3 P_{3 / 2}$ transition; irradiance: $4 \times 10^{4} \mathrm{~W} / \mathrm{cm}^{2}$; the $5 S-3 P$ transition served as fluorescence monitor. 


\subsection{Multiphoton Ionization Versus Collisional Ionization}

If three-photon ionization of the ground-state atoms by strong laser irradiation would be the dominant process, the quasistationary ion density would be expected to depend on the $3 / 2$ power of the laser power. This follows from Eqs. (2) to (6) where we have to replace the ionization rate constant by a factor that is proportional to the cube of laser power and $\left[\mathrm{Na}^{\phi}\right]$ by the $\mathrm{Na}$ ground-state density. Even with a fully saturated $3 S-3 P$ transition, the quasistationary ion density would depend linearly upon the laser power, as two laser photons are required to raise the atom from the saturated $3 P$ state to the continuum state. We observed (see Fig. 8) that the ionization signal with the laser tuned to the $3 S-3 P_{3 / 2}$ transition and the probe configuration of Fig. 1(a), did follow the square root of the laser power for low laser powers and tends to a constant value for higher laser powers. The latter behavior of the observed ionization signals reflects the behavior of the square root of the population density of the $3 P$ level, which acts as a quasi-ground level [cf., Eq. (6)] ; this population density is proportional to the laser power for low laser powers and saturates with increasing laser power. Besides, should multiphoton processes play an appreciable role, then the dependence of the degree of ionization on the free oxygen concentration as reported in Section 3.5 would be hard to understand.
We therefore conclude that the contribution of multiphoton processes to the observed ionization signals is negligible when the laser is tuned to the $3 S-3 P$ transition.

\subsection{Associative Ionization Versus Collisional Ionization}

In the case of saturation of the $3 P$ level, a possible ionization mechanism could be the associative reaction

$\mathrm{Na}(3 P)+\mathrm{Na}(3 P) \rightarrow \mathrm{Na}_{2}{ }^{+}+e^{-}$

which is about thermoneutral if $\mathrm{Na}_{2}{ }^{+}$is formed in the ground state. The effective cross section for this reaction is of the order of $5 \times 10^{-18} \mathrm{~cm}^{2}$ at a relative velocity of $4 \times 10^{4} \mathrm{~cm} \mathrm{sec}^{-1}$, as determined recently in an atomic beam experiment at our laboratory with laser-excited $\mathrm{Na}$ atoms in the $3 P$ level [26]. Using this cross-sectional value and the estimated (maximum) density of $\mathrm{Na}$ atoms in the $3 P$ level (about $10^{13} \mathrm{~cm}^{-3}$ ), one calculates that the rate of process (14) is less than the collisional ionization rate $R_{i}{ }^{3 P}$ calculated with the aid of Eq. (2), by two orders of magnitude. In the latter calculation, we have used the known thermal ionization rate constant, $k_{i}{ }^{0}(T)$, for ground-state $\mathrm{Na}$ atoms in similar flames at $T=1800 \mathrm{~K}$ [2] and the estimated ratio of the quasi-thermal rate constant $k_{i}{ }^{3 P}(T)$ to $k_{i}{ }^{0}(T)$, given by exp $\left[+E_{\text {exc }}{ }^{3 P}\right]$ $k T]$ (cf., Section 3.3).

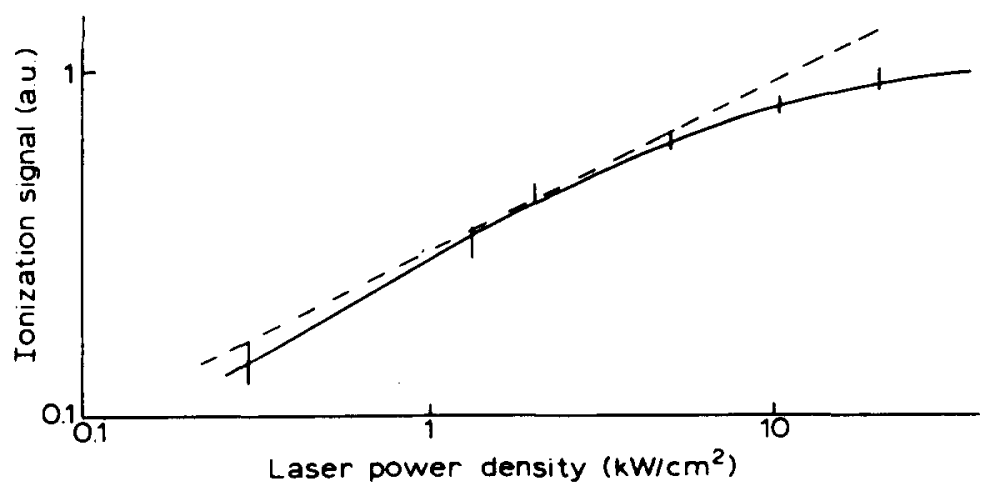

Fig. 8. Ionization signal as a function of the laser power on double-logarithmic scales. The laser was tuned to the $3 S-3 P_{3 / 2}$ transition and the probe configuration of Fig. 1(a) was used. The dashed line has a slope of 0.5 . 
Moreover, in the case of process (14), one would expect that the ionization signal, under quasistationary conditions, is not proportional to $\left[\mathrm{Na}^{3 P}\right]^{1 / 2}$ but to $\left[\mathrm{Na}^{3 P}\right]$, as $R_{i}{ }^{3 P}$ occurring in Eq. (2) would then be proportional to $\left[\mathrm{Na}^{3 P}\right]^{2}$. This expectation is at variance with the observed decline of the ionization signal per unit $\mathrm{Na}$ concentration with increasing $\mathrm{Na}$ concentration in Fig. 5(a). Finally, the marked dependence of the ionization signal on the free- $\mathrm{O}_{2}$ concentration (see Fig. 6) could then not be explained.

Therefore we conclude that associative ionization from the $3 P$ state did not play a competitive role in the laser-induced ionization under our experimental conditions.

\section{CONCLUSIONS}

Tuned to one- or two-photon transitions of the sodium atom, a laser, with a pulse duration of approximately $1 \mu \mathrm{sec}$ and irradiances ranging from $40 \mathrm{~kW} / \mathrm{cm}^{2}$ to $4 \times 10^{4} \mathrm{~kW} / \mathrm{cm}^{2}$, considerably enhances the ionization rate of these atoms in atmospheric-pressure flames of about $1800 \mathrm{~K}$. A pair of biased iridium probes proved to be a suitable means to detect the ionization in the small laser-irradiated region of the flame.

The larger width of the ionization profiles as compared to that of the fluorescence excitation profiles, obtained by detuning the laser, is explained qualitatively by the assumption of an effective Saha equilibrium during the laser pulse. This equilibrium is based on the balance between the collisional rate of ionization from the saturated $\mathrm{Na}$ level and the thermal collisional recombination rate. This effective Saha equilibrium may also explain the dependence of the ionization on the sodium solution concentration. From this dependence it follows that the degree of ionization is small for sodium solution concentrations in excess of $100 \mu \mathrm{g} /$ mliter approximately, corresponding to a total density of about $10^{12} \mathrm{Na}$ atoms per $\mathrm{cm}^{3}$ in the flame.

The presence of free $\mathrm{O}_{2}$ molecules in fuel-lean flames appears to shift the effective Saha equilibrium in the direction of increasing ionization degree, whereas the addition of $\mathrm{N}_{2}$ in comparable concentrations does not have this effect. The different effects of $\mathrm{O}_{2}$ and $\mathrm{N}_{2}$ can be qualitatively understood from the positive electron affinity of $\mathrm{O}_{2}$, contrasted with the negative electron affinity of $\mathrm{N}_{2}$.

When the laser is tuned to the $3 S-3 P$ transition, additional one-photon excitation in the collisionally broadened wing of the $3 P-5 S$ transition can explain why the ionization signal with the saturated $3 P$ level is much higher than would be expected from the activation energy factor for this level.

From the dependence of the ionization signal on the laser irradiance we conclude that the contribution of multiphoton ionization directly from the ground state or first excited state is negligible.

Also the contribution of associative ionization from the saturated $3 P$ level appears to be negligible in our flames; this outcome supports the crosssectional value for the latter process recently found in atomic beam experiments at our laboratory.

The authors are indebted to Dr. P. J. Th. Zeegers for his comments and reading of the manuscript.

\section{REFERENCES}

1. Dijk, C. A. van, Zeegers, P. J. Th., and Alkemade, C. Th. J., J.Q.S.R.T. 21, 115 (1979).

2. Hollander, Tj., Kalff, P. J., and Alkemade, C. Th. J., J. Chem. Phys. 39, 2558 (1963); Kelly, R., and Padley, P. J., Proc. Roy. Soc. London A 327, 345 (1972).

3. Ashton, A. F., and Hayhurst, A. N., Combust. Flame 21, 69 (1973).

4. Schott, L., in Plasma Diagnostics (W. Lochte Holtgreven, Ed.), North Holland, Amsterdam, The Netherlands, 1968, ch. 11, p. 711.

5. Green, R. B., Keller, R. A., Schenck, P. K., Travis, J. C., and Luther, G. G., J.Am. Chem. Soc. 98, 8517 (1976).

6. Green, R. B., Keller, R. A., Luther, G. G., Schenck, P. K., and Travis, J. C., Appl. Phys. Lett, 29, 727 (1976).

7. Green, R. B., Keller, R. A., Luther, G. G., Schenck, P. K., and Travis, J. C., IEEE J. Quantum Electron. QE-13, 63 (1977).

8. Alkemade, C. Th. J., Proc. 20th Coll. Spectrosc. Int. and 7th Int. Conf. on Atomic Spectrosc., Prague, 1977, "Invited lectures," Sbornik VSCHT, Prague, 1977 , p. 93.

9. King, D. S., and Schenck, P. K., Laser Focus 14, 50 (1978).

10. Dijk, C. A. van, Zeegers, P. J. Th., and Alkemade, C. Th. J., J.Q.S.R.T. 20, 55 (1978).

11. Kuhl, J., Marowsky, G., Kunstmann,P., and Schmidt, W., Zs. Naturforsch. 27a, 601 (1972). 
12. Behmenburg, W., J.Q.S.R.T. 4, 177 (1964).

13. Lijnse, P. L., and Elsenaar, R. J., J.Q.S.R.T. 12, 1115 (1972).

14. Wilson, H. A., Rev. Mod. Phys. 3, 156 (1931).

15. Calcar, R. A. van, Ven, M. J. M. van de, Uitert, B. K. van, Biewenga, K. J., Hollander, Tj., and Alkemade, C. Th. J., J.Q.S.R.T. 21, 11 (1979).

16. Hollander, $\mathrm{Tj} .$, Thesis, Utrecht, 1964.

17. Gallagher, T. F., Edelstein, S. A., and Hill, R. M., Phys. Rev. A 15, 1945 (1977).

18. Humphrey, L. M., Gallagher, T. F., Cooke, W. E., and Edelstein, S. A., Phys. Rev. A 18, 1383 (1978).

19. Gounand, F., Fournier, P. R., and Berlande, J., Phys. Rev. A 15, 2212 (1977).

20. Gounand, F., Cuvellier, J., Fournier, P. R., and Berlande, J., J. Physique 37, L-169 (1976).

21. Smirnov, B. M., Invited Lectures, Review Papers and Progress Reports of the Ninth International Confer- ence on the Physics of Electronic and Atomic Collisions (T. S. Risley and R. Geballe, Eds.), University of Washington Press, 1975.

22. Snelleman, W., Thesis, Utrecht, 1965; Ashton, A. F., and Hayhurst, A. N., Trans. Faraday Soc. 66, 833 (1970).

23. McDaniel, E. W., Collision Phenomena in Ionized Gases, Wiley, New York, 1964, p. 493.

24. Baede, A. P. M., in Advances in Chemical Physics (K. P. Lawley, Ed.), Wiley, New York-London, 1975, vol. 30 , p. 463.

25. Dijk, C. A. van, Thesis, Utrecht, 1978 (available on request).

26. Jong, A. de, and Valk, F, van der, J. Phys. B, 12, L-561 (1979).

Received 22 May 1979; revised 29 August 1979 